\title{
Network And Meta-Omics Reveal The Key Degrader And Cooperation Patterns In An Efficient 1,4-Dioxane-Degrading Microbial Community
}

Chuhan Dai

Zhejiang University

Hao Wu

Zhejiang University

\section{Xuejun Wang}

Zhejiang University

Kankan Zhao

Zhejiang University

Zhenmei Lv ( $\sim$ Izhenmei@zju.edu.cn )

Zhejiang University https://orcid.org/0000-0003-4768-9522

\section{Research}

Keywords: 1,4-Dioxane, Microbial community, Meta-omics, Network, Cooperation, Biodegradation

Posted Date: September 20th, 2021

DOI: https://doi.org/10.21203/rs.3.rs-877942/v1

License: (c) (i) This work is licensed under a Creative Commons Attribution 4.0 International License. Read Full License 


\section{Abstract}

Background: 1,4-dioxane is an emerging wastewater contaminant with probable human carcinogenicity. Our current understanding of microbial interactions during 1,4-dioxane biodegradation process in mix cultures is limited. Here, we applied metagenomic, metatranscriptomic and co-occurrence network analyses to unraveling the microbial cooperation between degrader and non-degraders in an efficient 1,4-dioxane-degrading microbial community $\mathrm{CH}$.

Results: The 1,4-dioxane degrading bacterium, Ancylobacter polymorphus ZM13, was isolated from $\mathrm{CH} 1$ and proved to be the key degrader because of the high relative abundance, highly expressed toluene monooxygenase genes $t m o A B C D E F$ and high betweenness centrality of networks. The strain ZM13 cooperated obviously with 6 bacterial genera in the network, among which Xanthobacter and Mesorhizobium were proved to be involved in the intermediate metabolism with responsible genes encoding alcohol dehydrogenase $(a d h)$, aldehyde dehydrogenase (aldh), glycolate oxidase ( $g / c D E F)$, glyoxylate carboligase $(g c l)$, malate synthase $(g / c B)$ and 2-isopropylmalate synthase (leuA) upregulated. Also, 1,4-dioxane facilitated the shift of biodiversity and function of $\mathrm{CH} 1$, and those cooperators of $\mathrm{CH} 1$ cooperated with $\mathrm{ZM} 13$ in the way of providing amino acids or fatty acids and relieving environmental stresses to promote biodegradation.

Conclusions: This study revealed the biodiversity, community structure, microbial functions and interactions in a microbial community $\mathrm{CH} 1$ during the efficient 1,4-dioxane degradation and proved the degrader Ancylobacter polymorphus ZM13 that isolated from $\mathrm{CH} 1$ was the key degrading bacterium. These results provide new insights into our understanding of how the key degrading bacterium interacted with cooperators in a 1,4-dioxane-degrading community, and has important implications for predicting microbial cooperation and constructing highly efficient synthetic 1,4-dioxane-degrading communities.

\section{Background}

In nature, microorganisms live in the form of communities and interact with each other in different ways, such as commensalism and mutualism [1]. Interactions help microorganisms resist environmental disturbances and accomplish tasks that a single microbe could not achieve. In remediation process, cooperators help improve the degrading efficiency by transferring toxic intermediates to less toxic ones [2], secreting growth or degradation stimulating substances [3] or by relieving the environmental stresses [4]. Costa et al. and Mao et al., respectively, have illustrated the functional diversity in a microbial polymer-degrading microbial community [5] and an enhanced biological phosphorus removal sludge [6] using the combination of metagenomics and other technologies. Moreover, the microbial co-occurrence patterns, functions as well as the driving mechanisms in different habitats, including soil [7], forests [8] and fresh water [9] were successfully predicted by co-occurrence network analysis, which is based on the abundance information provided by 16S rRNA gene amplicon sequencing. Statistically significant associations between different nodes in the resulting network can be the guidance of identifying probable cooperative bacteria. Recent studies have proved the feasibility of predictions based on networks with the isolation of previously uncultivated bacteria [10] and cooperative bacteria for pollutant degradation [11].

1,4-dioxane is widely used as a solvent or stabilizer in the industrial production and is easy to accumulate in groundwater, surface water and even drinking water [12]. Due to its probable human carcinogenicity, the U.S. Environmental Protection Agency (USEPA) has put 1,4-dioxane on the list of "high priority" pollutants. Compared with traditional physicochemical remediation methods, microbial remediation is recognized as a more economical and safe method to deal with contaminants. Since the first 1,4-dioxane-degrading strain Rhodococcus ruber 219 was isolated [13], a number of degrading isolates have been reported. The best characterized 1,4-dioxane degrader Pseudonocardia dioxanivorans CB1190 is a Grampositive strain with a specific degradation rate of $1.98 \mathrm{mg}$ dioxane $\mathrm{h}^{-1} \mathrm{mg}_{\text {protein }}{ }^{-1}$ [14], and has been widely studied and applied in a laboratory [15] or field [16] scale. The biodegradation pathways of 1,4-dioxane were studied in different strains. Soluble di-iron monooxygenases (SDIMOs) have been reported as the primary enzymes that initiate 1,4-dioxane degradation by converting 1,4-dioxane to 2-hydroxy-1,4-dioxane [17]. Grostern et al. proved that genes related to glycolate metabolism as well as aldehyde dehydrogenase and alcohol dehydrogenase genes were upregulated in CB1190 during 1,4-dioxane degradation using a microarray, which contributed to the understanding of 1,4-dioxane downstream metabolism [18]. 
However, most strains have limited specificity and degradation efficiency, or can only co-metabolite 1,4-dioxane with the existence of another substrate that induces degradation enzymes, such as propane [19] and tetrahydrofuran [20].

To date, there is no report of specific bacterial cooperation that enhanced 1,4-dixoane degradation. Yet, a thiamine-mediated cooperation for the degradation of tetrahydrofuran (a structural analog and co-metabolic substrate of 1,4-dioxane) has been verified [21]. The potential degraders and main cooperators in the degradation of tetrahydrofuran were also identified by metagenomic analysis [22]. A recent study discovered potential novel pathways and enzymes for 1,4-dioxane degradation using the integration of metagenomics and metabolomics [23]. Nevertheless, the application of amplicon sequencing and network analysis in understanding the microbial interactions for 1,4-dioxane degradation is limited. Most studies only gave overall descriptions of 1,4-dixaone degradation, including key functional enzymes [24], microbial responses, or microbial interactions with the existence of co-contaminants [25].

In this study, a mix community $\mathrm{CH} 1$ that efficiently degraded 1,4-dioxane was enriched from activated sludge, and a degrading bacterium was isolated from $\mathrm{CH} 1$ and identified as Ancylobacter polymorphus ZM13. Amplicon sequencing, metagenomics and metatranscriptomics as well as the basic monitoring of the culture were applied throughout a microcosm experiment to explore 1) the structure and function change of $\mathrm{CH} 1$ when removing high concentrations of 1,4dioxane as well as the relevant degrading enzymes; 2) the role of the degrader ZM13 in 1,4-dioxane degradation; 3 ) the microbial interaction patterns that support the efficient 1,4-dioxane degradation and potential cooperation mechanisms between ZM13 and cooperators. These findings provide a basis for further understanding microbial interactions and degradation mechanisms in the process of 1,4-dioxane remediation and have important implications for constructing synthetic microbial consortia for efficient 1,4-dioxane remediation.

\section{Methods}

\section{Chemicals, media and growth conditions}

1,4-dioxane (99.0\%, AR grade) and other chemical reagents used in the bacterial culture were purchased from the Sinopharm Chemical Reagent (Shanghai, China) and Aladdin Bio-Chem (Shanghai, China). Basal salts medium (BSM) was used for enrichment and cultivation. One liter of BSM contained $100 \mathrm{~mL}$ of BSM buffer stock and $100 \mathrm{~mL}$ of BSM trace metal stock. The BSM buffer stock (per liter) contained $32.4 \mathrm{~g}$ of $\mathrm{K}_{2} \mathrm{HPO}_{4}, 10.0 \mathrm{~g}$ of $\mathrm{NaH}_{2} \mathrm{PO}_{4} \cdot \mathrm{H}_{2} \mathrm{O}$ and $20.0 \mathrm{~g}$ of $\mathrm{NH}_{4} \mathrm{Cl}$. The $\mathrm{BSM}$ trace metal solution (per liter) contained $1.23 \mathrm{~g}$ of disodium nitrilotriacetate, $2.0 \mathrm{~g} \mathrm{MgSO} \cdot 7 \mathrm{H}_{2} \mathrm{O}, 0.12 \mathrm{~g} \mathrm{FeSO} \cdot 7 \mathrm{H}_{2} \mathrm{O}$, $0.03 \mathrm{~g} \mathrm{MnSO}_{4} \cdot \mathrm{H}_{2} \mathrm{O}, 0.03 \mathrm{~g} \mathrm{ZnSO}_{4} \cdot 7 \mathrm{H}_{2} \mathrm{O}$, and $0.01 \mathrm{~g} \mathrm{CoCl}_{2} \cdot 6 \mathrm{H}_{2} \mathrm{O}$. All media were autoclaved at $121^{\circ} \mathrm{C}$ for $15 \mathrm{~min}$ before used. All cultures were incubated at $30{ }^{\circ} \mathrm{C}$ with $180 \mathrm{rpm}$ agitation and inoculated at an initial optical density at $600 \mathrm{~nm}\left(\mathrm{OD}_{600}\right)$ of 0.05 .

\section{Culture enrichment and isolation of bacteria}

The highly efficient 1,4-dioxane-degrading microbial community $\mathrm{CH} 1$ used in this study was enriched from the activated sludge that collected from the sewage treatment site with long term 1,4-dioxane contamination of a 1,4-dioxanemanufacturing plant (Jiangsu, China). Ten $\mathrm{mL}$ of the sludge was inoculated into a 500-mL flask containing $100 \mathrm{~mL}$ of BSM medium amended with 1,4-dioxane as the sole carbon source. The acclimation and enrichment of the culture lasted for one month with 1,4-dixane concentration ranging from 1 to $12 \mathrm{mmol} / \mathrm{L}(\mathrm{mM})$. Every three days, the precedent enrichment culture was washed by BSM medium and sub-cultured to fresh BSM medium with a higher concentration of 1,4-dioxane added. After incubation and acclimatization, the culture $\mathrm{CH} 1$ was capable of completely degrading $12 \mathrm{mM}$ 1,4-dioxane in three days and could be stably sub-cultured. Degrading experiments with different initial 1,4-dioxane concentrations $(20,50,90,110$, $130,160,190,210 \mathrm{mM}$ ) were performed to assess the tolerance concentration of $\mathrm{CH} 1$ and the impacts of initial 1,4-dioxane concentration on the degradation. The methods of kinetic model used to fit the degradation data are presented in Additional file 1: Supplementary methods. 
In order to isolate 1,4-dioxane-degrading and non-degrading bacteria from $\mathrm{CH} 1$, the culture sample of each generation was collected and plated on BSM agar amended with 1,4-dioxane, LB agar or selected media based on the later network analysis. The colonies were picked and inoculated into BSM liquid medium amended with 1,4-dioxane to examine the ability of 1,4dioxane degradation. The identification was performed by sequencing the amplified 16S rRNA genes.

\section{Microcosm experiment}

A microcosm experiment was constructed to study the degradation capability of $\mathrm{CH} 1$ and the impact of high levels of 1,4dioxane contamination on the microbial community. The enrichment culture was collected, centrifuged and washed with BSM for three times. After resuspended with BSM, 1\% (v/v) of the mother liquor was inoculated into $150 \mathrm{~mL}$ of BSM medium. To extend the monitoring time, $60 \mathrm{mM}$ of 1,4-dioxane was added to each microcosm. The microcosms were kept until 1,4-dioxane concentration was below the detection level. Culture samples were collected every 24 hours. One part of the samples was used for measuring the growth, 1,4-dioxane concentration and $\mathrm{pH}$ value. Another part was stored at $-80^{\circ} \mathrm{C}$ for later DNA or RNA extraction. Treatment amended with same concentration of sodium succinate but without 1,4-dioxane was taken as a control for high-throughput sequencing. The experiment was performed with five independent biological replicates.

\section{Analysis of 1,4-dioxane concentrations}

Concentrations of 1,4-dioxane were monitored by GC-2014C gas chromatography equipped with a Flame lonization Detector (FID) and an AOC-20i Auto injector (SHIMADZU, Shanghai, China). The samples were centrifuged at 10,000 rpm for $1 \mathrm{~min}$ and the supernatant was filtered with a $0.22-\mu \mathrm{m}$ filter to remove the bacteria. The aqueous samples $(1 \mu \mathrm{L})$ were directly injected by an automatic injection. The temperatures of injector and detector were set at $250{ }^{\circ} \mathrm{C}$ and $300{ }^{\circ} \mathrm{C}$, respectively. The initial temperature of oven was set at $60^{\circ} \mathrm{C}$ and gradually increased to $160{ }^{\circ} \mathrm{C}$ at a rate of $20^{\circ} \mathrm{C} / \mathrm{min}$, and maintained for $1 \mathrm{~min}$.

\section{DNA, RNA extraction and sequencing of 16S rRNA gene amplicons, metagenomics and metatranscriptomics}

In the experiment amended with 1,4-dioxane, bacterial samples for 16S rRNA gene amplicon sequencing were collected every 24 hours with 1.5-mL centrifuge tubes (total 9 sampling time points). Three DNA samples and three RNA samples used for metagenomic and metatranscriptomic sequencing were collected from $50 \mathrm{~mL}$ of culture samples at day 4 , respectively. Another three RNA samples used as a control for metatranscriptomic sequencing were collected from the culture samples supplemented with sodium succinate. Details of the collected DNA and RNA samples are listed in Additional file 1: Table S1.

Total DNA and RNA extraction was carried out with a bacterial DNA and RNA kit (Omega Bio-Tek, GA, USA), respectively following the manufacture's instructions. The quality and quantity of the extracts were tested by the NanoDrop One (Thermo Fisher Scientific, MA, USA) and agarose gel electrophoresis. All sequencing tasks were performed at the Magigene Biotech (Guangzhou, China).

For 16S rRNA gene amplicon sequencing, the V3-V4 regions of 16S rRNA genes were amplified with primer sets 341F (5'CCTACGGRRBGCASCAGKVRVGAAT-3') and 806R (5'-GGACTACNVGGGTWTCTAATCC-3') and purified using an E.Z.N.A. Gel Extraction Kit (Omega, USA). The library was constructed and sequenced on the platform of Illumina Nova6000 (IIlumina, CA, USA).

For metagenomic and metatranscriptomic sequencing, the fragment library of DNA and cDNA was generated according to the protocol with specific index codes added. After quality assessment and cluster generation, the library was sequenced on a high-throughput sequencing platform of Illumina with 150-bp paired-end reads.

\section{Microbial community analysis}


The operational taxonomic units (OTUs) were clustered from the sequence reads at a similarity level of more than $99 \%$ using UPARSE pipeline [26]. Normalized OTU table was generated based on the sample with the least sequences. For each OTU, a representative sequence was selected for the annotation of the species. STAMP software (version 2.1.3)

(https://beikolab.cs.dal.ca/software/STAMP), and R software (version 3.6.3) (http://www.r-project.org/) were used to perform the statistics analyses and species community structure analyses.

To analyze a-diversity of different samples, complexity of species diversity was evaluated by calculating indices including Chao 1, Pielou, Shannon, etc. $p$ values were calculated by the Student's t-test to evaluate the significant differences between different groups. Least significance difference (LSD) analysis was used for multiple comparisons. $\beta$-diversity between samples of different time points was analyzed by principal coordinates analysis (PCOA) and displayed by the vegan package in R software [27]. The methods of LEfSe analysis were presented in Additional file 1: Supplementary methods.

\section{Molecular ecological network analysis}

Molecular ecological network analysis was conducted based on random matrix theory (RMT) methods [28]. The Spearman correlation matrix was calculated by $\mathrm{R}$ software according to the relative abundances of OTUs in each sample. To reduce the bias of correlation coefficients, OTUs with relative abundances less than 72 were not considered for generating the network. The cutoff $p$ values of correlation adjusted with false discovery rate (FDR) [29] was 0.05 . The cutoff value of correlation coefficients was 0.66. R packages, including vegan, Hmisc [30] and igraph [31] were used in the above analysis. The visualization of the co-occurrence networks and the detection of modules were conducted by Gephi software [32]. Each node in the network represents an OTU. The edges connect every two nodes indicate positive or negative correlations of OTUs. All the $\mathrm{R}$ codes used for network constructing referred to a previous study [33].

\section{Metagenomic and metatranscriptomic analysis}

The de novo mixed assembly of metagenomic reads was carried out with MEGAHIT [34] to obtain scaffolds. Scaffolds were then interrupted from $\mathrm{N}$ connection to obtain scaftigs without $\mathrm{N}$ and fragments shorter than $2500 \mathrm{bp}$ were filtered out. After assembly, the clean data of each sample was mapped to the scaffolds by Bowtie2 [35] to calculate the depth. Binning was conducted using MetaBAT2 (version 2.15) (https://bitbucket.org/berkeleylab/metabat) according to the read coverage, coverage variance and tetranucleotide frequencies. After quality assessment, genomes with high completeness and low contamination were selected as good bins. For open reading frame (ORF) prediction, MetaGeneMark (version 3.38) (http://exon.gatech.edu) and CD-HIT (version 4.7) (http://www.bioinformatics.org/cd-hit) were used. The abundance of genes was calculated based on the number of mapped reads and the length of genes. ORFs were further blasted to functional databases including KEGG, NR and EggNOG with an evalue of $1 \mathrm{e}^{-10}$ for functional annotations. Phylogenetic analysis was conducted using GTDBTk (https://github.com/Ecogenomics/GtdbTk) based on genome taxonomy database (GTDB) to determine the species classification of genomes.

Metatranscriptomic reads were assembled using the Trinity software (version 2.4.0) (http://trinityrnaseq.github.io) and redundancy was eliminated using CD-HIT with an identity of $95 \%$ and coverage of $90 \%$ to obtain the genes. The read count of each gene was obtained from the mapping results and the RPKM value was calculated to estimate the gene expression levels [36]. The predicted genes were blasted to different databases including GO, KEGG, CAZy and EggNOG by BLASTx for the annotation of species and functions with an evalue of $1 \mathrm{e}^{-5}$. Gene expressions and KEGG pathways were visualized using heat maps by R software.

\section{Results}

\section{Community $\mathrm{CH} 1$ degraded 1,4-dioxane efficiently and a degrader ZM13 was isolated from $\mathrm{CH} 1$}


The microbial community $\mathrm{CH} 1$ enriched in this study had a highly efficient 1,4-dioxane degradation capability, which could degrade more than $98 \%$ of $50 \mathrm{mM}$ 1,4-dioxane within 6 days (Additional file 1: Figure S1). With the increase of 1,4-dioxane concentrations, the growth rate initially increased and then decreased, which fitted the Haldane's growth model well (Additional file 1: Figure S2) and the tolerance concentration was up to $190 \mathrm{mM}$ (Additional file 1: Figure S1a). The

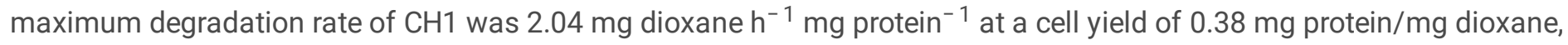

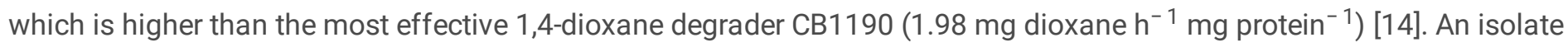
that could utilize 1,4-dioxane as sole carbon and energy source was isolated from $\mathrm{CH} 1$, and was identified as Ancylobacter polymorphus and designated as ZM13 (Additional file 1: Figure S3).

Based on the degradation performances of $\mathrm{CH} 1$ at different concentrations of 1,4-dioxane (Additional file 1: Figure S1), we selected a high initial 1,4-dioxane concentration of $60 \mathrm{mM}$ to conduct the microcosm experiment with five biological replicates for high consistency, which also showed enough experimental time for sampling. $\mathrm{CH} 1$ removed total $60 \mathrm{mM} \mathrm{1,4-}$ dioxane to below detection levels within 8 days with a short and unobvious lag phase while 1,4-dioxane concentration in the abiotic control did not decline (Fig. 1a). Considering acidic intermediates that were continuously produced during 1,4dioxane degradation, $\mathrm{pH}$ value was measured. As a result, the $\mathrm{pH}$ remained stable in the first two days and sharply decreased with a massive degradation of 1,4-dioxane (Fig. 1b), indicating the accumulation of acidic metabolites.

\section{The bacterial community was affected by the addition of 1,4-dioxane and tended to stabilize with 1,4-dioxane exhaustion}

To assess the change of community composition and structure during 1,4-dioxane degradation, sequencing of 16S rRNA gene amplicons was performed with a total of 13,356,940 raw reads obtained. The raw reads were then clustered at a similarity level of $99 \%$ and a total of 358 OTUs were obtained. a-diversity analysis showed that the bacterial diversity significantly $(p<0.05)$ increased right after the addition of 1,4-dioxane (Fig. 2a, b). Results of PCoA indicated that the samples collected from day 0 separated significantly $(p<0.05)$ with others (Fig. $2 \mathrm{c})$, which is consistent with a-diversity analysis, and the separation degree gradually decreased with time and finally tended to be consistent. These results indicated that the high concentrations of 1,4-dioxane had a substantial impact on $\mathrm{CH} 1$, and the structure tended to stabilize with the exhaustion of 1,4-dioxane.

The taxonomy results were shown in Fig. $2 \mathrm{~d}$ and Additional file 1: Figure S4. The composition of $\mathrm{CH} 1$ remained relatively stable during the microcosm experiment. The community was dominated by the phylum Proteobacteria and Alphaproteobacteria was the most abundant class (Additional file 1: Figure S4). Class Betaproteobacteria had a sharp increase on day 2 and gradually decreased from day 4, meanwhile class Gammaproteobacteria began to increase (Additional file 1: Figure S4). Abundances of some dominant genera had a significant $(p<0.05)$ change along with the 1,4dioxane degradation processes (LSD, Additional file 1: Table S2). The relative abundance of Achromobacter sharply increased to $26.32 \%$ at day 1 and gradually decreased to $2.71 \%$ (Fig. 2 d). Oligotropha was the dominant genus during the whole experiment while other dominant genera switched from Sphingopyxis (21.77-10.77\%) to Aquamicrobium (6.63$18.23 \%$ ) (Fig. 2d). The isolated 1,4-dioxane degrader, A. polymorphus, kept at a relative abundance of $0.5 \%-2 \%$ and its dynamic change seemed to be most consistent with Xanthobacter (LSD, Additional file 1: Table S2), indicating a potential cooperation between A. polymorphus and Xanthobacter. Moreover, the results of LEfSe analysis were presented in Additional file 1: Supplementary results and Figure S5.

\section{Co-occurrence network analysis revealed a cooperation between ZM13 and 6 bacterial genera for 1,4-dioxane degradation}

To investigate the interactions between ZM13 and other community members in $\mathrm{CH} 1$, OTUs from day 0 to day 8 were used to construct co-occurrence networks and the global network properties were listed in Additional file 1: Table S3. Totally, 113 
of 358 OTUs were presented in the output network with strong (Spearman's $\rho>0.66)$ and significant $(p<0.05)$ correlations, which generally indicated the co-occurrence patterns and the sharing niches were shaped by 1,4-dioxane degradation.

The network was divided into 7 modules with a good modularity index of 0.509 , of which three distinct key modules, module $\nabla, \nabla$ and $\otimes$, had OTUs with strong correlations with each other (Fig. 3a). The number of positive correlations (red edges) far exceeded that of negative correlations (blue edges) with a ratio of 81.83 (Fig. 3b), which implied a strong microbial cooperation outweighed competition during 1,4-dioxane degradation. Most of the negative correlations observed were between module $\otimes$ and module $\otimes$ (Fig. 3a). Also, OTUs belonged to Achromobacter, Sphingopyxis, Oligotropha, Rhodanobacter, Mersorhizobium, Alicycliphilus, and Hydrogenophaga had positive correlations with pH, while OTUs belonged to Aquamicrobium, Xanthomonas, Mesorhizobium, Afipia, Moheibacter, Lysobacter, Thermomonas, and Rhodopseudomonas had negative correlations with $\mathrm{pH}$ (Additional file 1: Figure S6). These results indicated that negative correlations might be caused by the microbial response to $\mathrm{pH}$ change during 1,4-dioxane degradation.

Although class Alphaproteobacteria had an overwhelming proportion to other class, Betaproteobacteria was the dominant class in the network (Additional file 1: Figure S7). Furthermore, the hubs (nodes with highest degree) were dominated by Betaproteobacteria while the bottlenecks (nodes with highest betweenness) were dominated by Alphaproteobacteria (Additional file 1: Table S4 and Table S5). Most of the hubs were identified as Achromobacter and the top 10 hubs were all

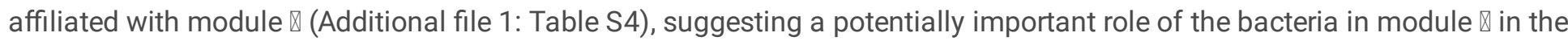
community assembly.

The 1,4-dioxane degrader ZM13 (OTU14) located in the position that connected the modules and did not seem to have a tight connection to any of the key modules in the network with only 7 correlations to other OTUs (Fig. 3b). A subnetwork centered on ZM13 was constructed to explore possible connections between ZM13 and other bacteria in the community (Fig. 3c). Specifically, ZM13 had positive correlations with Aquamicrobium, Mesorhizobium, Sphingopyxis, Xanthobacter, Cohnella and Achromobacter, and negative correlations with Pleomorphomonas (Additional file 1: Table S6). Nevertheless, ZM13 was recognized as one of the top-scoring bottlenecks located in the core of the network because of a high betweenness centrality of 873.55 (Additional file 1: Table S5). These results indicated that ZM13 was a keystone species in the biological network.

\section{Identification of 1,4-dioxane-degradation-related genes in individual microbes by the combination of metagenomic and metatranscriptomic analyses}

To investigate the metabolic capability and activity of individual microbes in microbial community $\mathrm{CH} 1$ during 1,4-dioxane degradation, an integrated analysis of metagenomic and metatranscriptomic data was applied with a total of $277,554,584$ raw reads obtained from metagenomic sequencing and 540,603,556 raw reads obtained from metatranscriptomic sequencing.

We recovered 43 bins from metagenomic assemblies, from which four high-quality bins with high abundance in the community as well as high genome completeness (>95\%) and low genetic contamination $(<2 \%)$ were selected with the potential involvement in 1,4-dioxane degradation for further phylogenetic analysis and functional annotation (Table 1). The four acquired genomes were identified as genera Sphingopyxis, Mesorhizobium, Achromobacter and Xanthobacter, respectively, and a total of $87.68-89.97 \%$ of predicted ORFs of the four strains were identified using the RAST Server (Rapid Annotation using Subsystem Technology) (Table 1). 
Table 1

Genomic information of species obtained from binning.

\begin{tabular}{|lllllllll|}
\hline Bins & $\begin{array}{l}\text { GTDB } \\
\text { annotation }\end{array}$ & $\begin{array}{l}\text { Genome } \\
\text { size } \\
\text { (Mbp) }\end{array}$ & GC\% & $\begin{array}{l}\text { Completeness } \\
(\%)\end{array}$ & $\begin{array}{l}\text { Contamination } \\
(\%)\end{array}$ & $\begin{array}{l}\text { No. of } \\
\text { Scaffolds }\end{array}$ & $\begin{array}{l}\text { No. } \\
\text { of } \\
\text { ORFs }\end{array}$ & $\begin{array}{l}\text { Annotation } \\
\text { ratio (\%) }\end{array}$ \\
\hline 1 & Sphingopyxis & 3.43 & 65.27 & 99.3 & 1.76 & 12 & 3328 & 89.97 \\
\hline 2 & Mesorhizobium & 4.55 & 63.43 & 96.55 & 1.78 & 131 & 4670 & 88.03 \\
\hline 3 & Achromobacter & 6.73 & 67.32 & 98.6 & 0.57 & 56 & 6208 & 87.58 \\
\hline 4 & Xanthobacter & 4.82 & 67.96 & 95.22 & 0.32 & 24 & 4678 & 88.68 \\
\hline
\end{tabular}

Non-redundant gene catalogue (Unigenes) containing a total number of 297,123 genes was obtained from the predicted ORFs of metatranscriptomic sequencing with the total length of $179,797,590 \mathrm{bp}$. The overall descriptions of KEGG pathways during 1,4-dioxane degradation were presented in Additional file 1: Supplementary results and Figure S8. Further analyses were focused on the genes encoding enzymes that were possibly involved in 1,4-dioxane degradation, including monooxygenase genes, 1,4-dioxane metabolism intermediate-related genes, and glycolate and glyoxylate metabolismrelated genes [18] that were expressed in ZM13 and the acquired bins. For the genome sequencing method and genome information of ZM13, see Additional file 1: Supplementary methods and Supplementary results.

All the differentially expressed genes potentially involved in 1,4-dioxane degradation were displayed in heat map and mapped to the genome of each strain (Additional file 1: Figure S9). The proposed 1,4-dioxane degradation pathway reported previously were shown in Additional file 1: Figure S10. The results indicated that $\mathrm{CH} 1$ possessed the genes responsible for glyoxylate metabolism pathways for 1,4-dioxane downstream metabolism (Additional file 1: Figure S9). The top expressed monooxygenase genes were all identified as toluene monooxygenase genes and were unique in ZM13 with high identities ranging from 97-100\% (Additional file 1: Table S7 and Figure S9a). Moreover, ZM13 contained complete pathways for 1,4dioxane degradation (Fig. 4a, b). These results indicated that ZM13 was a key degrader in 1,4-dioxane degradation.

The integrated meta-omics analysis revealed that although Mesorhizobium and Xanthobacter lacked monooxygenase genes for the first step of degradation, they contained upregulated genes for the degradation of 1,4-dioxane downstream metabolites (Fig. 4b, c, d and Additional file 1: Figure S9). Mesorhizobium lacked the genes responsible for converting 2hydroxy-1,4-dioxane to 2-hydroxyethoxyacetic acid (2HEAA) (aldh), glycoaldehyde to glycolic acid (aldh) and glyoxylic acid to tartronate semialdehyde $(g c l)$, but contained genes responsible for converting glycolate to malate $(g / c D, g / c E, g / c F, g / c B$, leuA) (Fig. 4b, c, d). Genes of aldh and gc/ were only identified once in ZM13 and Xanthobacter, which could suggest that the dehydrogenation of aldehydes and the glyoxylate carboligase pathway might not be the main pathway for 1,4-dioxane degradation.

In conclusion, these results provided the evidence that $A$. polymorphus ZM13 was the key degrader that converted 1,4dioxane to 2-hydroxy-1,4-dioxane and other intermediates that supported the growth of non-degrading populations, such as Mesorhizobium and Xanthobacter.

\section{Discussion}

Compared with a single microbe, a microbial consortium contains more genes with stronger metabolic capacity, allowing for the ability to survive environmental perturbation and the division of labor for pollutant remediation [37]. The microbial community $\mathrm{CH} 1$ enriched in this study with high efficiency for 1,4-dioxane removal and the key degrader ZM13 isolated from $\mathrm{CH} 1$ by traditional methods were investigated to reveal the community dynamics, metabolic activity, microbial interactions and possible cooperation mechanisms during 1,4-dioxane degradation.

\section{The isolated degrading bacterium ZM13 played the key degrading function in $\mathrm{CH} 1$}


Ancylobacter is mainly known as facultative methylotroph that could use metsulfuron-methyl or 1,2-dichloroethane [38] as sole carbon and energy source. This is the first time of this genus to be reported to have 1,4-dioxane-degrading ability. The reported 1,4-dioxane degraders mostly belong to Gram-positive bacteria. Among Gram-negative strains, such

as Xanthobacter flavus DT8 [39] and Azoarcus sp. DD4 [19] that can metabolize or co-metabolize 1,4-dioxane, ZM13 had a closer relationship with DT8 (Phylogenetic tree, Additional file 1: Figure S3).

The community $\mathrm{CH} 1$ exhibited much better degrading performance than the single degrader ZM13. Before the investigation of microbial interactions in $\mathrm{CH} 1$, it is of great importance to identify the degrading role of ZM13 in the community. Yu et al., for instance, had successfully identified the key degraders in a bisphenol A-degrading microbial community using the combination of meta-omics technologies [40]. In this study, we confirmed ZM13 as the key degrading bacterium in different ways. 16S rRNA gene sequencing revealed that ZM13 was one of the dominate species in $\mathrm{CH} 1$ during the mineralization process of 1,4-dioxane, though without an overwhelming proportion (Fig. 2d). Metatranscriptomic sequencing revealed that among the multiple monooxygenase genes detected in the culture, including toluene monooxygenase, 4-

hydroxyphenylacetate 3-monooxygenase, nitronate monooxygenase, salicylate 1-monooxygenase and alkanesulfonate monooxygenase genes, the most abundant monooxygenase genes from ZM13 were identified as toluene monooxygenase

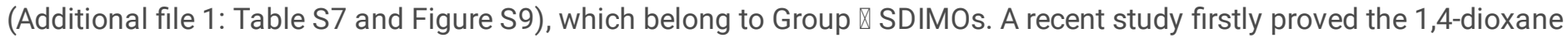
degradation function of toluene monooxygenase in a propanotrophic strain DD4 [41]. In addition, the network analysis demonstrated the important role of ZM13 as a bottleneck, serving as a bridge that connected different parts of the community. A bottleneck is the node with high betweenness centrality and betweenness represents the number of the shortest paths between any other two nodes that pass through that node [42]. Moreover, LEfSe analysis also indicated that ZM13 played an important role at the beginning of the 1,4-dioxane degradation (Additional file 1: Supplementary results and Figure S5). These evidences strongly supported that ZM13 could play the key degrading role and might act as an organizer in $\mathrm{CH} 1$ during the mineralization process.

\section{1,4-dioxane shaped the biodiversity and functional shift of $\mathrm{CH} 1$}

The $\mathrm{CH} 1$ community structure was reshaped under the continuous stress of 1,4-dioxane. The biodiversity analysis indicated that the addition of 1,4-dioxane did increase the biodiversity although the community was relatively stable. The dominant genera of $\mathrm{CH} 1$ included Oligotropha, Sphingopyxis, Achomobacer, Xanthobacter, and Aquamicrobium, which were similar with a previously enriched 1,4-dioxane-degrading consortium [23]. The relatively stable structure of $\mathrm{CH} 1$ during 1,4-dioxane degradation indicated the complex and stable synergistic effect in the community and strains with high abundance other than ZM13 might either contributed to the degradation or acted as cheaters [43].

As a toxic compound, 1,4-dioxane could cause the stress responses of the microbial community and change microbial interactions and functions [44]. In this study, the present of 1,4-dioxane upregulated KEGG pathways of bacterial secretion systems, quorum sensing components and $A B C$ transporters which all played important roles in the adaptation to environmental disturbances (Additional file 1: Figure S8). Bacterial secretion system is a versatile weapon that helps microorganism interactions and is mediated by the secretion, diffusion and exchange of diverse molecules [45], and quorum sensing is a density-dependent form of communication that involves the production of and response to these molecules [46]. ABC transporters were reported to have the ability to improve bacterial acid tolerance [47]. Environmental pH is one of the key factors affecting microbial growth. As reported, many acidic metabolites, such as 2HEAA, glycolic acid and glyoxylic acid [18], are continuously produced and accumulated during the biodegradation of 1,4-dioxane, leading to the huge drop of $\mathrm{pH}$ values. Moreover, genes that associated with several well-studied anti-acid systems were identified, including genes responsible for arginine/glutamate decarboxylation [48], and exopolysaccharide production [49] (Additional file 1: Table S8). These results demonstrated that the addition of 1,4-dioxane facilitated the shift of community function and enriched genes adaptable to microenvironmental changes and stresses.

\section{Network analysis and integrated analysis of metagenomics and metatranscriptomics revealed consistent microbial interactions}

Page 9/18 
Co-occurrence network analysis indicated that $\mathrm{CH} 1$ was a microbial community with high positive internal correlations during 1,4-dioxane degradation. Six genera were found to be positively correlated with ZM13 in the network, among which the genomic information of Sphingopyxis, Mesorhizobium, Achromobacter and Xanthobacter were acquired and the genes related to 1,4-dioxane degradation in the recovered genomes were revealed. The genus Achromobacter was reported to regulate its own population density through quorum sensing [50], which might explain its high abundance in the community and high degree centrality in the network (Additional file 1: Table S5). The genus Sphingopyxis has been reported to possess degrading capabilities for various environmental contaminants [51]. However, these two genera did not contribute much to 1,4-dioxane degradation directly as few related genes were discovered (Additional file 1: Figure S9).

On the contrary, Xanthobacter and Mesorhizobium possessed quite a lot upregulated genes that were related to 1,4-dioxane degradation (Additional file 1: Table S7 and Figure S9). The degrading genes identified in Xanthobacter and Mesorhizobium provided an evidence of potential substrate cross-feeding and solidly supported the correlations predicted by network analysis. A cooperative cross-feeding between degraders and non-degraders was common in the degradation of many organic compounds [40]. Downstream metabolites including glycolic acid, ethylene glycol and malic acid were identified by GC-MS during 1,4-dioxane degradation in this study, and ZM13 was verified to have utilization capacity of these intermediates (Additional file 1: Figure S11). The differentially upregulated gene numbers in Xanthobacter and Mesorhizobium suggested the metabolic division of labor during 1,4-dioxane degradation. The functional shift and metabolites exchange, as well as the diversity of metabolic pathways possessed by different community members enable the community to efficiently cycle available nutrients and to survive environmental perturbations or stresses [52].

\section{Possible mechanisms of cooperation between degrader ZM13 and its cooperators}

Overall, the above results indicated that although the key degrading bacteria are essential for biodegradation, community members involved in intermediate degradation and environmental adaptation are also indispensable for the efficient degradation. Genera Xanthobacter and Mesorhizobium have been identified in multiple consortia that had 1,4-dioxanedegrading capability $[23,53]$, suggesting potentially important roles of these two bacteria in the cooperation of 1,4-dioxane degradation. However, because of the slow growth rate of Mesorhizobium even at the optimum medium [54] and the difficulty of finding the optimum medium for cultivating Xanthobacter, as well as the tight interspecies collaboration in the community, these two strains were not successfully isolated. Nevertheless, using KEGG Mapper tools (https://www.kegg.jp/kegg/mapper/), we discovered the complementarity of metabolic pathways between ZM13 and Xanthobacter or Mesorhizobium, including the synthetic pathways of arginine, histidine, and fatty acids (Additional file 1: Figure S12). The exchanges of metabolites, such as amino acids, sugars, and fatty acids, are important cooperative interactions that improve efficiency of resource utilization and provide a survival advantage for the community members [55]. Such metabolites exchanges have been confirmed in both microbial communities [56] and co-cultures of two strains [57]. The experiment of arginine supplementation verified that the addition of arginine could improve the degrading efficiency of ZM13 (Additional file 1: Figure S13).

Based on the above results, we proposed a microbial cooperation model between degrader ZM13 and its cooperators in the community $\mathrm{CH} 1$ (Fig. 5): ZM13 initiated the degradation of 1,4-dioxane with toluene monooxygenase and generated intermediates, which could be consumed by intermediate-utilizers, such as Xanthobacter and Mesorhizobium who possessed the intermediate-degrading genes; in return, the cooperators provided nutrients such as amino acids and fatty acids and provided protection for environmental disturbances and $\mathrm{pH}$ stress to accelerate the degradation process of 1,4dioxane.

\section{Conclusions}

In summary, this study revealed the biodiversity, community structure, microbial functions and interactions in a microbial community $\mathrm{CH} 1$ during the efficient 1,4-dioxane degradation and proved the degrader Ancylobacter polymorphus ZM13 that isolated from $\mathrm{CH} 1$ was the key degrading bacterium. With the help of meta-omics tools, the possible metabolites exchange

Page $10 / 18$ 
between ZM13 and two intermediate-utilizing bacteria Xanthobacter and Mesorhizobium were described. Furthermore, the microbial co-occurrence patterns during 1,4-dioxane degradation by the combination of network, metagenomic and metatranscriptomic analysis proved the feasibility of predicting bacterial correlations. We also proposed a conceptual model to describe possible cooperation patterns between the degrader and cooperators in $\mathrm{CH} 1$. These findings are not only meaningful for understanding of adaptation mechanisms of 1,4-dioxane-degrading consortia exposed to 1,4-dioxane and how community members perform interspecies collaboration but also helpful for the future bioremediation applications of 1,4-dioxane-contaminated sites.

\section{Abbreviations}

tmoABCDEF: toluene monooxygenase; adh: alcohol dehydrogenase; aldh: aldehyde dehydrogenase; glcDEF: glycolate oxidase; gcl: glyoxylate carboligase; glcB: malate synthase; leuA: 2-isopropylmalate synthase; SDIMOs: Soluble di-iron monooxygenases; BSM: Basal salts medium; 2HEAA: 2-hydroxyethoxyacetic acid.

\section{Declarations}

\section{Acknowledgments}

We acknowledge the members in Jiangsu runlian Chemical Co., Ltd (Jiangsu, China) for their assistance in the sampling of activated sludge.

\section{Authors' contributions}

ZL, CD and XW conceived and designed the study. KZ contributed in the analysis of the 16S rRNA gene amplicon sequencing data. HW contributed in the analysis of the metagenomic and metatranscriptomic data. CD wrote the manuscript drafts and all other authors revised the manuscript. All authors read and approved the final manuscript.

\section{Funding}

This work was financially supported by grants from the National Natural Science Foundation of China (Nos. 41630637, 41721001, and 32170107).

\section{Availability of data and material}

Raw reads of $16 \mathrm{~S}$ rRNA gene sequencing were deposited into NCBI Sequence Read Archive (SRA) under the accession number PRJNA759367. Raw reads of metagenomic and metatranscriptomic sequencing, the genomes of Ancylobacter polymorphus ZM13 and the metagenomic-assembled bins were deposited into NCBI SRA under the accession number PRJNA759764. The 16S rRNA genes of Ancylobacter polymorphus ZM13 were deposited into GenBank databases under accession numbers OK021564.

\section{Ethics approval and consent to participate}

Not applicable.

\section{Competing interests}

The authors declare that they have no competing interests.

\section{Consent for publication}

Not applicable. 


\section{References}

1. Bhatia SK, Bhatia RK, Choi Y-K, Kan E, Kim Y-G, Yang Y-H. Biotechnological potential of microbial consortia and future perspectives. Crit Rev Biotechnol. 2018;38(8):1209-29.

2. Seo H, Kim J, Jung J, Jin HM, Jeon CO, Park W. Complexity of cell-cell interactions between Pseudomonas sp. AS1 and Acinetobacter oleivorans DR1: metabolic commensalism, biofilm formation and quorum quenching. Res Microbiol. 2012;163(3):173-81.

3. Chen B-Y, Chen S-Y, Lin M-Y, Chang J-S. Exploring bioaugmentation strategies for azo-dye decolorization using a mixed consortium of Pseudomonas luteola and Escherichia coli. Process Biochem. 2006;41(7):1574-81.

4. Liu Z, He Z, Huang H, Ran X, Oluwafunmilayo AO, Lu Z. pH Stress-induced cooperation between Rhodococcus ruber YYL and Bacillus cereus MLY1 in biodegradation of tetrahydrofuran. Front Microbiol. 2017;8:2297.

5. Costa OYA, de Hollander M, Pijl A, Liu B, Kuramae EE. Cultivation-independent and cultivation-dependent metagenomes reveal genetic and enzymatic potential of microbial community involved in the degradation of a complex microbial polymer. Microbiome. 2020;8(1):76.

6. Mao Y, Yu K, Xia Y, Chao Y, Zhang T. Genome reconstruction and gene expression of "Candidatus Accumulibacter phosphatis" Clade IB performing biological phosphorus removal. Environ Sci Technol. 2014;48(17):10363-71.

7. Ma B, Wang HZ, Dsouza M, Lou J, He Y, Dai ZM, Brookes PC, Xu JM, Gilbert JA. Geographic patterns of co-occurrence network topological features for soil microbiota at continental scale in eastern China. ISME J. 2016;10(8):1891-901.

8. Tu QC, Yan QY, Deng Y, Michaletz ST, Buzzard V, Weiser MD, Waide R, Ning DL, Wu LY, He ZL, Zhou JZ. Biogeographic patterns of microbial co-occurrence ecological networks in six American forests. Soil Biol Biochem. 2020;148:107897.

9. Mikhailov IS, Zakharova YR, Bukin YS, Galachyants YP, Petrova DP, Sakirko MV, Likhoshway YV. Co-occurrence networks among bacteria and microbial eukaryotes of lake baikal during a spring phytoplankton bloom. Microb Ecol. 2019;77(1):96-109.

10. Xian W-D, Salam N, Li M-M, Zhou E-M, Yin Y-R, Liu Z-T, Ming Y-Z, Zhang X-T, Wu G, Liu L, Xiao M, Jiang H-C, Li W-J. Network-directed efficient isolation of previously uncultivated Chloroflexi and related bacteria in hot spring microbial mats. NPJ Biofilms Microbi. 2020;6(1):20.

11. Wang X, Wu H, Wang X, Wang H, Zhao K, Ma B, Lu Z. Network-directed isolation of the cooperator Pseudomonas aeruginosa ZM03 enhanced the dibutyl phthalate degradation capacity of Arthrobacter nicotianae ZM05 under pH stress. J Hazard Mater. 2021;410:124667.

12. Godri Pollitt KJ, Kim JH, Peccia J, Elimelech M, Zhang Y, Charkoftaki G, Hodges B, Zucker I, Huang H, Deziel NC, Murphy K, Ishii M, Johnson CH, Boissevain A, O'Keefe E, Anastas PT, Orlicky D, Thompson DC, Vasiliou V. 1,4-Dioxane as an emerging water contaminant: State of the science and evaluation of research needs. Sci Total Environ. 2019;690:85366.

13. Bernhardt D, Diekmann H. Degradation of dioxane, tetrahydrofuran and other cyclic ethers by an environmental Rhodococcus strain. Appl Microbiol Biotechnol. 1991;36(1):120-3.

14. Parales RE, Adamus JE, White N, May HD. Degradation of 1,4-dioxane by an actinomycete in pure culture. Appl Environ Microbiol. 1995;60(12):4527-30.

15. Barajas-Rodriguez FJ, Freedman DL. Aerobic biodegradation kinetics for 1,4-dioxane under metabolic and cometabolic conditions. J Hazard Mater. 2018;350:180-8.

16. Myers MA, Johnson NW, Marin EZ, Pornwongthong P, Liu Y, Gedalanga PB, Mahendra S. Abiotic and bioaugmented granular activated carbon for the treatment of 1,4-dioxane-contaminated water. Environ Pollut. 2018;240:916-24.

17. Li M, Mathieu J, Yang Y, Fiorenza S, Deng Y, He Z, Zhou J, Alvarez PJJ. Widespread distribution of soluble di-iron monooxygenase (SDIMO) genes in arctic groundwater impacted by 1,4-dioxane. Environ Sci Technol. 2013;47(17):9950-8.

Page $12 / 18$ 
18. Grostern A, Sales CM, Zhuang W-Q, Erbilgin O, Alvarez-Cohen L. Glyoxylate metabolism is a key feature of the metabolic degradation of 1,4-dioxane by Pseudonocardia dioxanivorans strain CB1190. Appl Environ Microbiol. 2012;78(9):3298308.

19. Deng D, Li F, Wu C, Li M. Synchronic biotransformation of 1,4-dioxane and 1,1-dichloroethylene by a Gram-negative propanotroph Azoarcus sp. DD4. Environ Sci Technol. 2018;5(8):526-32.

20. Vainberg S, McClay K, Masuda H, Root D, Condee C, Zylstra GJ, Steffan RJ. Biodegradation of ether pollutants by Pseudonocardia sp. strain ENV478. Appl Environ Microbiol. 2006;72(8):5218-24.

21. Huang H, Qi M, Liu Y, Wang H, Wang X, Qiu Y, Lu Z. Thiamine-mediated cooperation between auxotrophic Rhodococcus ruber ZM07 and Escherichia coli K12 drivesefficient tetrahydrofuran degradation. Front Microbiol. 2020;11:594052.

22. Qi M, Huang H, Zhang Y, Wang H, Li H, Lu Z. Novel tetrahydrofuran (THF) degradation-associated genes and cooperation patterns of a THF-degrading microbial community as revealed by metagenomic. Chemosphere. 2019;231:173-83.

23. Chen R, Miao Y, Liu Y, Zhang L, Zhong M, Adams JM, Dong Y, Mahendra S. Identification of novel 1,4-dioxane degraders and related genes from activated sludge by taxonomic and functional gene sequence analysis. J Hazard Mater. 2021;412(5):125157.

24. Guan X, Liu F, Wang J, Li C, Zheng X. Mechanism of 1,4-dioxane microbial degradation revealed by $16 S$ rRNA and metatranscriptomic analyses. Water Sci Technol. 2018;77(1):123-33.

25. Miao Y, Johnson NW, Phan T, Heck K, Gedalanga PB, Zheng X, Adamson D, Newell C, Wong MS, Mahendra S. Monitoring, assessment, and prediction of microbial shifts in coupled catalysis and biodegradation of 1,4-dioxane and co-contaminants. Water Res. 2020;173:115540.

26. Edgar RC. UPARSE: highly accurate OTU sequences from microbial amplicon reads. Nat Methods. 2013;10(10):996-8.

27. Dixon P. VEGAN, a package of R functions for community ecology. J Veg Sci. 2003;14(6):927-30.

28. Deng Y, Jiang Y-H, Yang Y, He Z, Luo F, Zhou J. Molecular ecological network analyses. BMC Bioinf. 2012;13:113.

29. Yekutieli DK. Adaptive linear step-up procedures that control the false discovery rate. Biometrika. 2006;93(3):491-507.

30. Jr Dupont F C. Hmisc: Harrell Miscellaneous. R. Package Version 3. 2008.

31. Csardi G, Nepusz T. The igraph software package for complex network research. J Interjournal Complex Systems. 2006;1695:1-9.

32. Bastian M, Heymann S, Jacomy M. Gephi. An open source software for exploring and manipulating networks. ICWSM. 2009;8:361-2.

33. Ju F, Xia Y, Guo F, Wang Z, Zhang T. Taxonomic relatedness shapes bacterial assembly in activated sludge of globally distributed wastewater treatment plants. Environ Microbiol. 2014;16(8):2421-32.

34. Li D, Liu C-M, Luo R, Sadakane K, Lam T-W. MEGAHIT: an ultra-fast single-node solution for large and complex metagenomics assembly via succinct de Bruijn graph. Bioinformatics. 2015;31(10):1674-6.

35. Langmead B, Salzberg SL. Fast gapped-read alignment with Bowtie 2. Nat Methods. 2012;9(4):357-9.

36. Wagner GP, Kin K, Lynch VJ. Measurement of mRNA abundance using RNA-seq data: RPKM measure is inconsistent among samples. Theor Biosci. 2012;131(4):281-5.

37. Hays SG, Patrick WG, Ziesack M, Oxman N, Silver PA. Better together: engineering and application of microbial symbioses. Curr Opin Biotechnol. 2015;36:40-9.

38. van den Wijngaard AJ, van der Kamp KW, van der Ploeg J, Pries F, Kazemier B, Janssen DB. Degradation of 1,2dichloroethane by Ancylobacter aquaticus and other facultative methylotrophs. Appl Environ Microbiol. 1992;58(3):976-83.

39. Chen D-Z, Jin X-J, Chen J, Ye J-X, Jiang N-X, Chen J-M. Intermediates and substrate interaction of 1,4-dioxane degradation by the effective metabolizer Xanthobacter flavus DT8. Int Biodeter Biodegr. 2016;106:133-40.

Page $13 / 18$ 
40. Yu K, Yi S, Li B, Guo F, Peng X, Wang Z, Wu Y, Alvarez-Cohen L, Zhang T. An integrated meta-omics approach reveals substrates involved in synergistic interactions in a bisphenol A (BPA)-degrading microbial community. Microbiome. 2019;7(1):16.

41. Deng D, Pham DN, Li F, Li M. Discovery of an inducible toluene monooxygenase that cooxidizes 1,4-dioxane and 1,1dichloroethylene in propanotrophic Azoarcus sp. strain DD4. Appl Environ Microbiol. 2020;86(17):e01163-01120.

42. Peura S, Bertilsson S, Jones RI, Eiler A. Resistant microbial cooccurrence patterns inferred by network topology. Appl Environ Microbiol. 2015;81(6):2090-7.

43. Feng $X$, Kostylev M, Dandekar AA, Greenberg EP. Dynamics of cheater invasion in a cooperating population of Pseudomonas aeruginosa. Sci Rep. 2019;9(1):10190.

44. Luo J, Zhang Q, Wu L, Cao J, Feng Q, Fang F, Chen Y. Inhibition of 1, 4-dioxane on the denitrification process by altering the viability and metabolic activity of Paracoccus denitrificans. Environ Sci Pollut Res Int. 2018;25(27):27274-82.

45. Johns NI, Blazejewski T, Gomes AL, Wang HH. Principles for designing synthetic microbial communities. Curr Opin Microbiol. 2016;31:146-53.

46. Mukherjee S, Bassler BL. Bacterial quorum sensing in complex and dynamically changing environments. Nat Rev Microbiol. 2019;17(6):371-82.

47. Zhu Z, Yang J, Yang P, Wu Z, Zhang J, Du G. Enhanced acid-stress tolerance in Lactococcus lactis NZ9000 by overexpression of ABC transporters. Microb Cell Fact. 2019;18(1):136.

48. Guan N, Liu L. Microbial response to acid stress: mechanisms and applications. Appl Microbiol Biotechnol. 2020;104(1):51-65.

49. Alp G, Aslim B. Relationship between the resistance to bile salts and low pH with exopolysaccharide (EPS) production of Bifidobacterium spp. isolated from infants feces and breast milk. Anaerobe. 2010;16(2):101-5.

50. Erandapurathukadumana Sreedharan H, Cherukara Chellappan H, Selvanesan P, Garvasis J. Quorum sensing mediated response of Achromobacter denitrificans SP1 towards prodigiosin production under phthalate stress. J Basic Microb. 2020;60(9):758-67.

51. Sharma M, Khurana H, Singh DN, Negi RK. The genus Sphingopyxis: Systematics, ecology, and bioremediation potential - A review. J Environ Manage. 2021;280:111744.

52. Che S, Men Y. Synthetic microbial consortia for biosynthesis and biodegradation: promises and challenges. $J$ Ind Microbiol Biotechnol. 2019;46(9-10):1343-58.

53. Ramalingam V, Cupples AM. Enrichment of novel Actinomycetales and the detection of monooxygenases during aerobic 1,4-dioxane biodegradation with uncontaminated and contaminated inocula. Appl Microbiol Biotechnol. 2020;104(5):2255-69.

54. Zhang JJ, Liu TY, Chen WF, Wang ET, Sui XH, Zhang XX, Li Y, Li Y, Chen WX. Mesorhizobium muleiense sp. nov., nodulating with Cicer arietinum L. Int J Syst Evol Microbiol. 2012;62(Pt 11):2737-42.

55. Zelezniak A, Andrejev S, Ponomarova O, Mende DR, Bork P, Patil KR. Metabolic dependencies drive species cooccurrence in diverse microbial communities. Proc Natl Acad Sci. 2015;112(20):6449-54.

56. Blasche S, Kim Y, Mars RAT, Machado D, Maansson M, Kafkia E, Milanese A, Zeller G, Teusink B, Nielsen J, Benes V, Neves R, Sauer U, Patil KR. Metabolic cooperation and spatiotemporal niche partitioning in a kefir microbial community. Nat Microbiol. 2021;6(2):196-208.

57. Wang X, Shen S, Wu H, Wang H, Wang L, Lu Z. Acinetobacter tandoii ZM06 assists Glutamicibacter nicotianae ZM05 in resisting cadmium pressure to preserve dipropyl phthalate biodegradation. Microorganisms. 2021;9(7):1417.

\section{Figures}


a

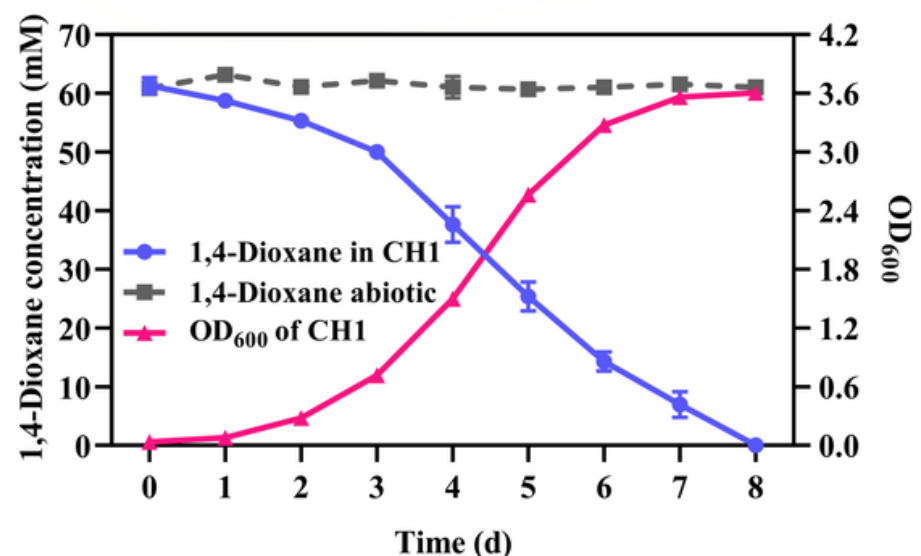

b

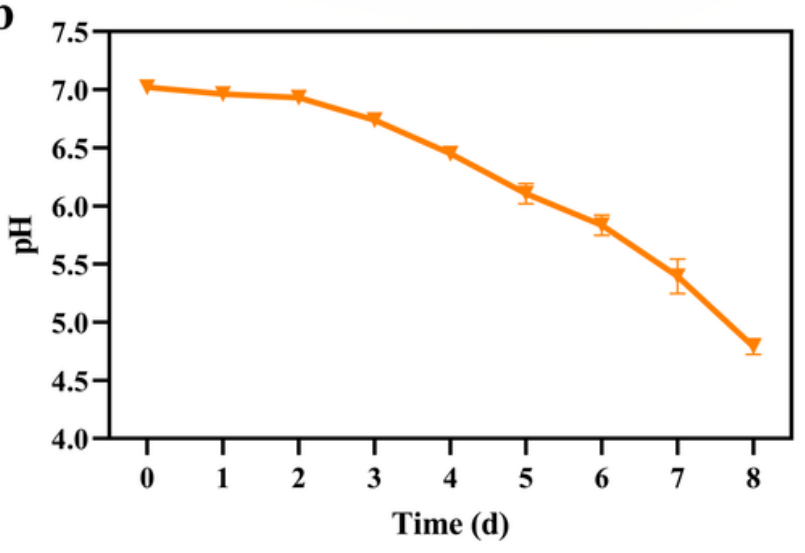

Figure 1

1,4-dioxane degradation (blue line), bacterial growth (red line) (a) and pH change (orange line) (b) of the enrichment culture during microcosm experiment. The grey dotted line in (a) represents the abiotic control to eliminate the effects of volatilization. Error bars represent the standard deviations of five replicates.

$\mathbf{a}$

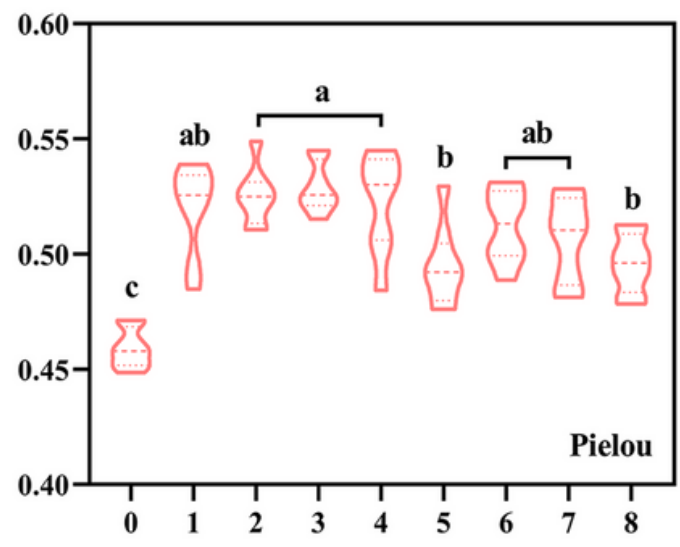

b

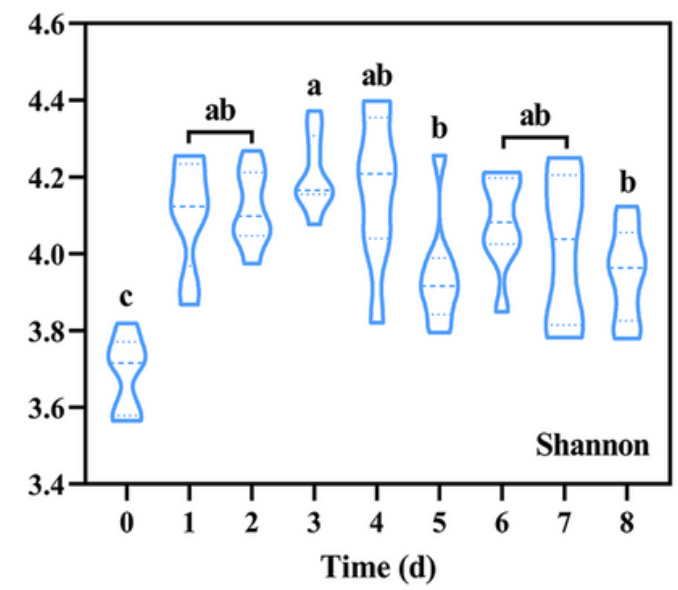

C

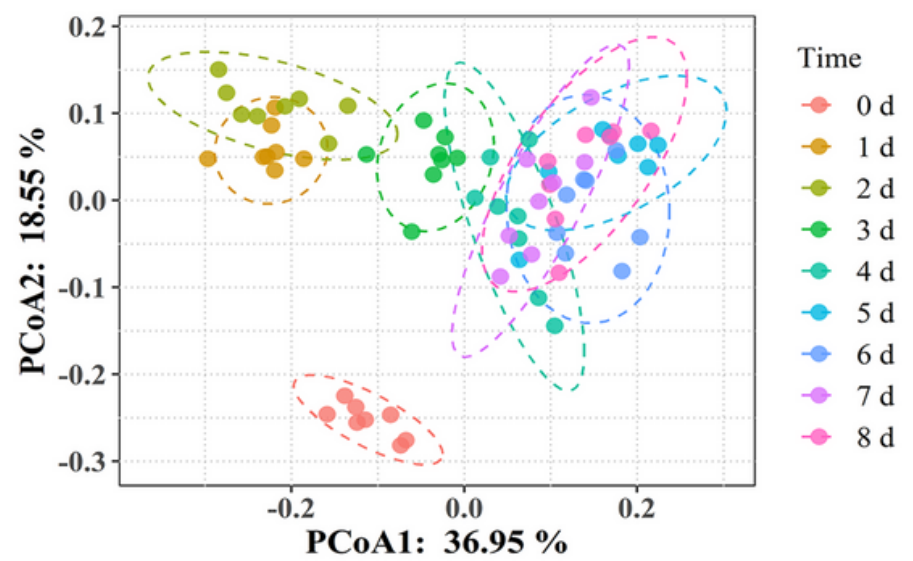

d

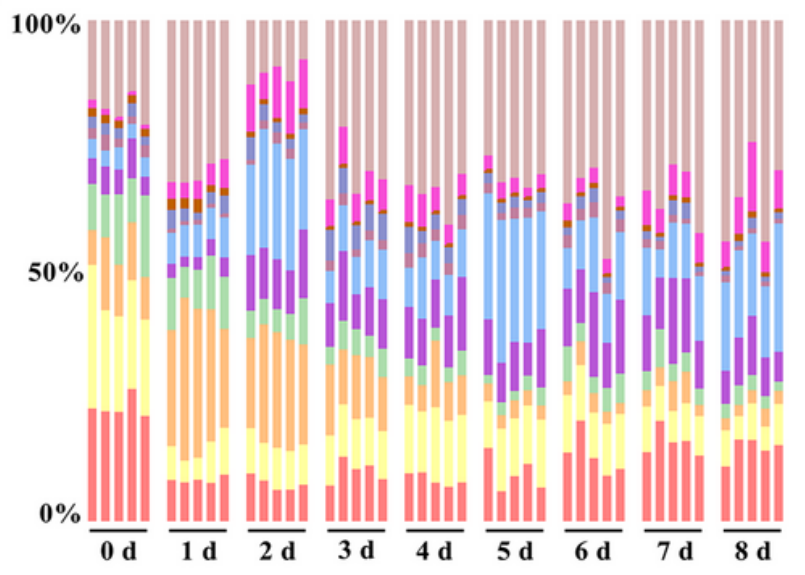

Others

Cohnella sp.

Ancylobacter sp.

Mesorhizobium sp.

Rhodanobacter sp.

Aquamicrobium sp.

Chelatococcus sp.

Xanthobacter sp.

Achromobacter sp.

Sphingopyxis sp.

Oligotropha sp.

\section{Figure 2}

Microbial community dynamics during 1,4-dioxane degradation. (a) Relative abundances of 16S rRNA gene sequence data at the genus level. Five replicates were shown at each time point; (b) Principal coordinate analysis (PCoA) at different 
sampling times; (c) The Pielou (red violins) and (d) Shannon (blue violins) indices of the microbial community at different sampling times. The different and same letters indicate significant $(p<0.05)$ and non-significant $(p>0.05)$ differences, respectively.

$\mathbf{a}$

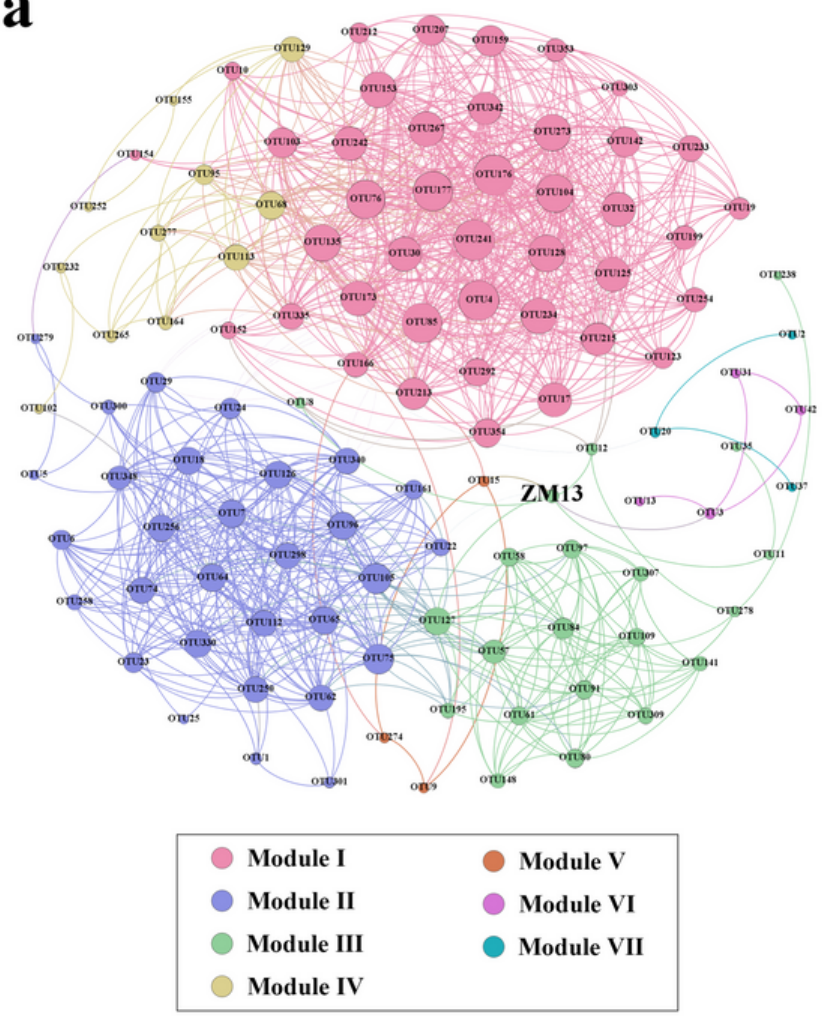

b

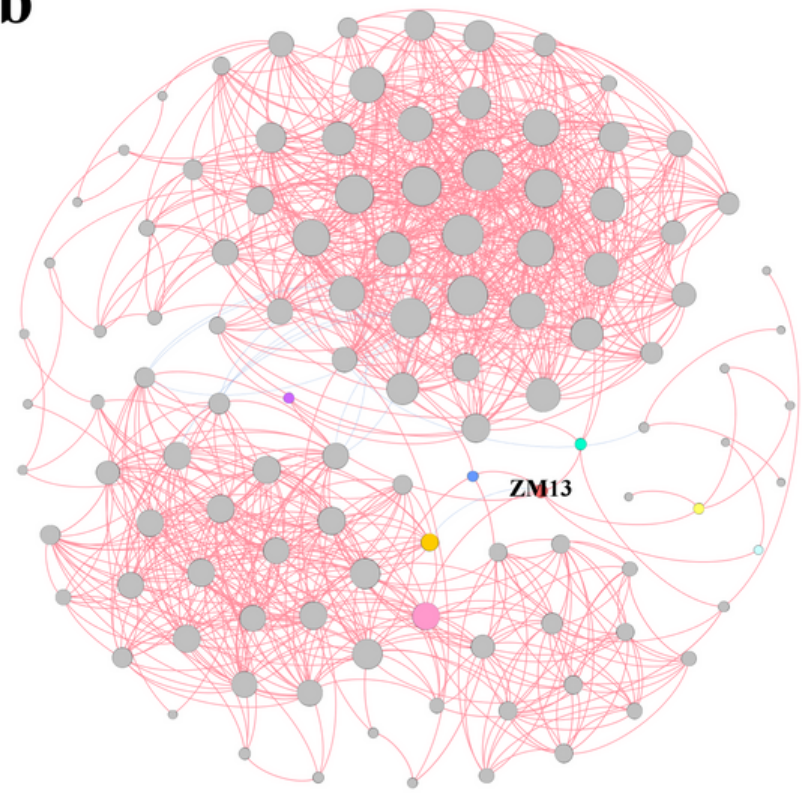

C

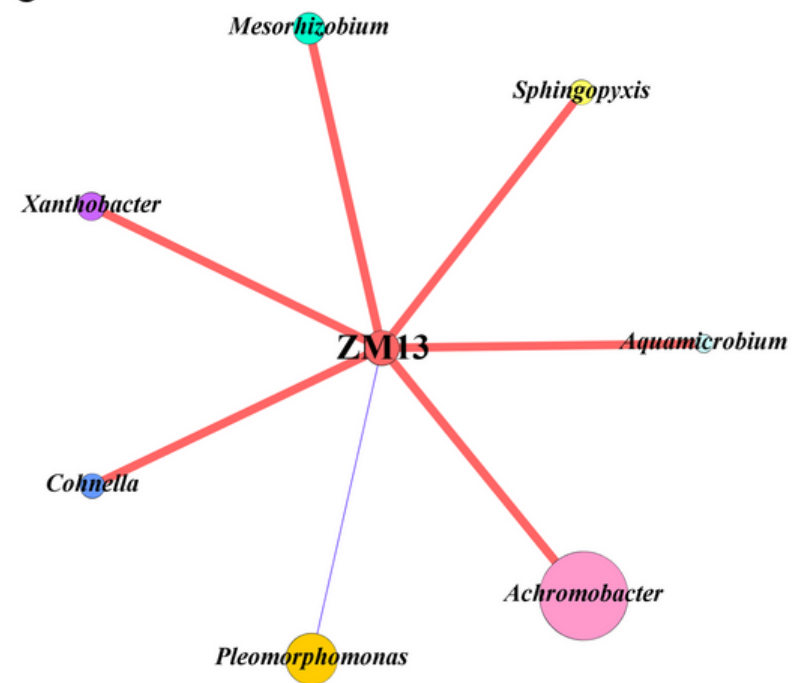

Figure 3

Co-occurrence network of OTUs based on correlation analysis. (a) Complete network colored by modules calculated by Gephi modularity toolkit. (b) Complete network displaying all nodes that connected to Ancylobacter polymorphurs ZM13 with different color. (c) Subnetwork of nodes connected to ZM13. The size of nodes is proportional to their degrees. Each edge represents a strong (Spearman's $\rho>0.66)$ and significant $(p<0.05)$ correlation. Red edges indicate positive correlations and blue edges indicate negative correlations. 

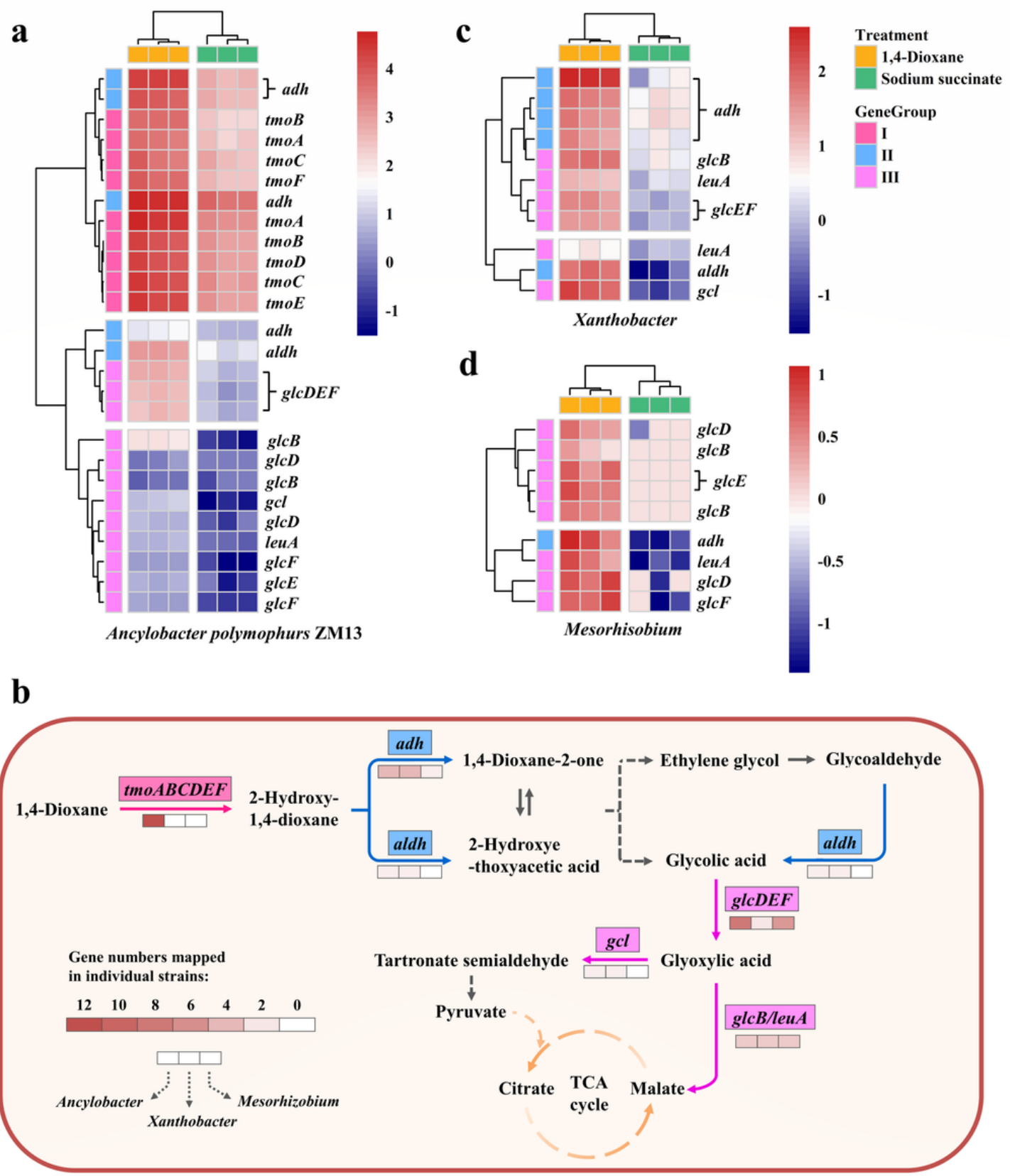

\section{Figure 4}

Heat map matrix of differential expression of genes ( $\mid$ Log2(Fold change) $\mid>1, p<0.05$ ) possibly involved in 1,4-dioxane degradation process that were identical to (a) A. polymophurs ZM13 (whole genome sequencing); (b) Xanthobacter (binning); (c) Mesorhizobium (binning). Gene group $\nabla$ (pink), $\otimes$ (blue) and $\otimes$ (purple) represents monooxygenase genes, 1,4dioxane metabolism intermediate-related genes and glycolate and glyoxylate metabolism-related genes. Treatments were cultured with 1,4-dioxane and sodium succinate respectively and three replicates were presented. (d) Pathway of 1,4-dioxane degradation with genes located in. tmoABCDEF, Toluene monooxygenase (EC:1.14.13.236; EC:1.18.1.3); adh, alcohol dehydrogenase (EC:1.1.1.-); aldh, aldehyde dehydrogenase, (EC:1.2.1.3); glcDEF, glycolate oxidase (EC:1.1.3.15); gcl, glyoxylate carboligase, (EC:4.1.1.47); glcB, malate synthase (EC:2.3.3.9); leuA, 2-isopropylmalate synthase (EC:2.3.3.13). Gene information is summarized in Additional file 1: Table S7. 


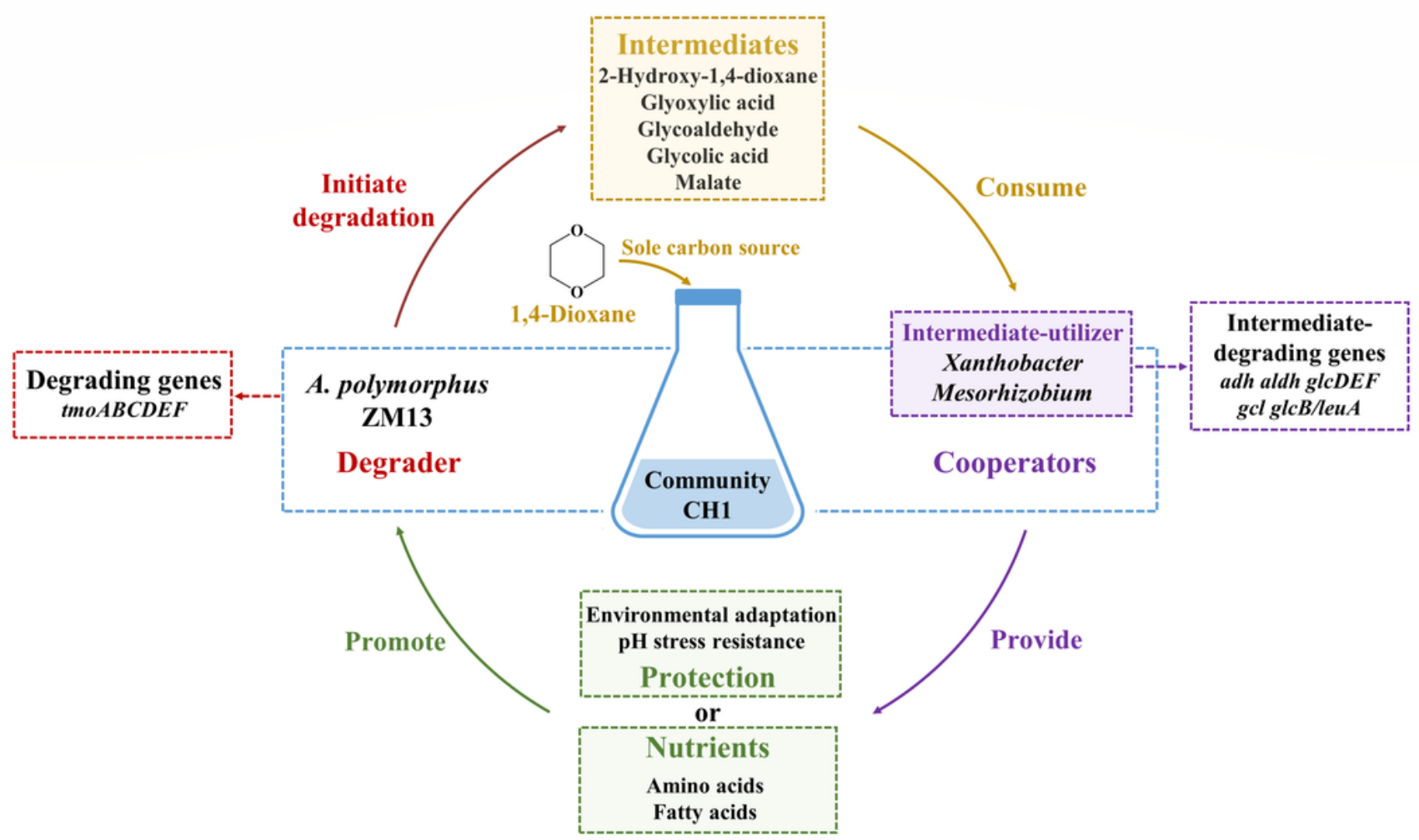

Figure 5

A proposed microbial cooperation model for 1,4-dioxane degrading strain ZM13 and its cooperators in the community $\mathrm{CH} 1$.

\section{Supplementary Files}

This is a list of supplementary files associated with this preprint. Click to download.

- Additionalfile1revised.docx 